\title{
Inhaltsverzeichnifs des sechs und vierzigsten Bandes, nach den Gegenständen.
}

\author{
I. Reine Mathematik.
}

Nr. der

Abhandlung.

1. A n a l y s i s.

1. I afeln für die Zerlegung der Zahlen bis 4100 in Biquadrate. Von Herrn Professor Dr. C. A. Bretschneider in Gotha. . . . . . . . . . . I. 1

5. Über die Verwandlung der Kettenbrüche in Reihen. Von dem Herrn Dr. Heilermann zu Trier.

7. Summen von Reihen, ausgedrückt durch bestimmte Integrale. Anwendungen dieser Sätze. Von Herrn Dr. Dienger, Professor der Mathematik an der polytechnischen Schule zu Carlsruhe im Badischen. . . . . . . .

10. Zur Theorie der elliptischen Functionen. Von Herrn R. Krusemarck, Cand. phil. zu Berlin.

II. 119

15. Über die Functionen, welche der Gleichung

$$
\varphi(x)+\varphi(y)=\psi\left(\frac{f y^{\prime} \cdot \boldsymbol{F} x+f x \cdot \boldsymbol{F y}}{\chi(x y)}\right)
$$

Genüge leisten. Von Herrn Dr. C. Lottner, Lehrer der Math. und Physik an der höheren Bürgerschule zu Lippstadt. . . . . . . . . . . . . . IV. 367

\section{G e o m e t r i e.}

8. Behandlung einiger Grund-Aufgaben der analytischen Geometrie, im schiefwinkligen Coordinatensystem. Von Herrn Dr. R. Baltzer, Oberlehrer am Gymnasio zu Dresden.

3. $\mathrm{M}$ e c h a n i k.

2. De la propriété fondamentale du mouvement cycloïdal, et de sa liaison avec le principe de la composition des mouvements de rolation autour d'axes parallèls et d'axes qui se coupent. Par Mr. Steichen, professeur à l'école militaire de Bruxelles.

3. Note sur le $\$$. 6 du mémoire No. 9 inséré dans le tome 43 de ce journal. Par le même auteur.

12. De orbitis et motibus puncti cuiusdam corporei circa centrum attractivum 13. aliis, quam Newtoniana, attractionis legibus sollicitati.

Ab Joh. Franc. Stader, stud. math. 
9. Beitrag zur Theorie der Bewegung der Räderfuhrwerke, mit Inbegriff der 11. Dampfwagen. Von Herrn J. P. G. von Heim, Königl. würtemb. Obrist14. lieutenant a. D.

12. De orbitis et motibus puncti cuiusdam corporei circa centrum attractivum 12. aliis, quam Newtoniana, attractionis legibus sollicitati. Ab Joh. Franc. 13. Stader, stud. math.

6. Lösung einiger Aufgaben aus der Axonometrie; mit besonderer Berücksichtigung der Anwendung derselben bei bildlicher Darstellung der Zwillingskrystalle. Von Herrn Gustav Zeuner, Berg-Ingenieur zu Chemnitz. .

Table d'errata, tome 43 , cahier 2 , pages 161 etc. . . . . . . . . . . $\quad$ I. 96 Druckfehlerverzeichnifs. 